\title{
BLOW UP OF MECHANICAL SYSTEMS WITH A HOMOGENEOUS ENERGY
}

\author{
ERnesto A. LACOMBA ${ }^{1}$, John BRyANT ANi LUis A. IBort ${ }^{2}$
}

\begin{abstract}
By using the ideas introduced by McGehee in the study of the singularities in some problems of Celestial Mechanics, we study the singularities at the origin and at the infinity for some classical mechanical systems with hornogeneous kinetic and potential energy functions. For these systems the origin and the infinity of the configuration coordinates is usually a singularity or a nullity of the Harniltonian function and the vector field. This work generalizes a previous one by the first and the third authors, where the kinetic energy did not depend on the configuration coordinates.
\end{abstract}

In this paper we study the blow up at the origin or at the infinity of configuration space in classical mechanical systems with homogeneous kinetic and potential energy functions. This means that usually the origin and the infinity of the configuration coordinates is a singularity or a nullity of the Hamiltonian function and the vector field. This is why a blow up of the function at infinity or at the origin has to be applied, which describes in some sense the asymptotic. behavior of escape orbits or of orbits going to the origin.

This approach was first applied to total collapse motion in celestial mechanics by McGehee [8]. It was then applied by several authors to total collapse of special 3 and 4-body problems (see the research notes [3], and references therein). Then the first author and Simó [7] generalized this procedure to the escape motion in celestial mechanics. An important feature of this generalization was that we had to consider 3 cases according to the energy sign, ard the case $h<0$ had to be treated differently, according to the specific problem. See the survey [4] and references therein.

The generalizations considered here for the case when the mass matrix is constant, were introduced in Lacomba and Ibort [5]. In a forthcoming paper the case of a non constant mass matrix $A$ will be treated in more detail.

${ }^{1}$ CIFMA member (Mexico).

${ }^{2}$ On leave of absence of Dept. of Fisica Teorica, Universidad Complutense de Madrid (Spain). 
An insight is made here into the nature of the blow up employed. We see that rather than projective we have a spherical blow up, where the radial coordinate is taken as non negative. This produces a fictitious boundary where the flow extends and is invariant. Under some conditions, the fow can be made projective but no boundary appears. All of this is discussed in Section 2.

In section 3 we give some important properties of the flow on the boundary manifolds. The presentation is given in a novel way with simpler proofs, although some results generalize [3] and others appear in [5]. Finally, the last two examples in Section 4 are analyzed here in this context for the first time.

\section{Statement of the problem and some examples}

The kind of homogeneous Hamiltonian functions considered here, are those of the form

$$
H(q, p)=\frac{1}{2} p A^{-1}(q) p^{t}-U(q)
$$

where $q$ belongs to the open cone $D \subset \mathbb{R}^{n}$ representing positions; $p \in \mathbb{R}^{n}$ are the momenta, roughly the duals of velocities; and $U: D \rightarrow \mathbb{R}$ is a homogeneous $C^{\infty}$ function of degree $k$ and $A: D \rightarrow M_{n}^{p d}(\mathbb{R})$ is homogeneous $C^{\infty}$ of degree $\alpha$, where $M_{n}^{p d}(\mathbb{R})$ is the space of positive definite $n \times n$ real matrices. This means that the first term in (1), known as the kinetic energy, is not only homogeneous (quadratic) in $p$, but also in $q$.

The corresponding Hamiltonian vector field is given by

$$
\begin{aligned}
& \dot{q}=\frac{\partial H}{\partial p}=p A(q)^{-1} \\
& \dot{p}=-\frac{\partial H}{\partial p}=\operatorname{grad} U(q)-\frac{1}{2} \frac{\partial}{\partial q}\left(p A^{-1}(q) p^{\ell}\right)
\end{aligned}
$$

If $A$ is constant, the second term in the equation for $\dot{p}$ does not appear, and the system is simpler.

As simple examples of this situation, we can mention the following ones.

(A) Total collision in $n$-body problems. In this case $k=-1, U$ is the gravitational potential among the $n$ bodies, and $A$ is a constant (the mass matrix). The origin $q=0$ correspond to total collapse of all the bodies, which is a singularity of $U$, and hence of the Harniltonian vector field. The blow up approach, valid for any energy was started by Mc Gehee for the collinear 3-body problem.

(B) Escape behavior in $n$-body problems. In the above problem we study a blow up at $q=\infty$, to describe escape motions. We have to distinguish the 3 cases $h>0, h=0$ or $h<0$, since the behavior and required transformations are quite different. 
(C) Conformal metrics of the form $d s^{2}=R^{\alpha}\left(d x_{1}^{2}+\cdots+d x_{n}^{2}\right)$ where $R^{2}=$ $x_{1}^{2}+x_{2}^{2}+\cdots+x_{n}^{2}$. In this case $R=0$ is a singularity or a degeneracy of the metric, according to the sign of $\alpha$.

(D) Hyperbolic half-plane. A variation of the above example is the hyperbolic metric $d s^{2}=y^{-2}\left(d x^{2}+d y^{2}\right)$ in the half-plane $y>0$. This corresponds to a case where $\alpha=-2$, and the boundary $y=0$ is a singularity of the metric.

In general, the Hamiltonian (1) has a singularity at $q=0$ and may go to zero at infinity if either $k<0$ or $\alpha>0$, exchanging behaviors at $q=0$ and at $q=\infty$ when $k>0$ or $\alpha<0$. In the next section we give a unified blow up treatment of this sort of behaviors.

\section{Blow up equations}

We essentially blow up the singularity of the Hamiltonian function, giving a corresponding resolution of the vector field.

Let $r=\left(q A(q) q^{t}\right)^{\frac{1}{\alpha+2}}$ for $\alpha \neq-2$ be a sort of "distance" to the origin, with a. "unit sphere"

$$
S=\left\{Q \in D \subset \mathbb{R}^{n}: Q A(Q) Q^{t}=1\right\},
$$

which may not be convex nor bounded in general. It is a $C^{\infty}$ manifold if $\alpha+2 \neq 0$. It is actually a unit sphere if $A$ is constant. For example, if $Q=(X, Y)$ and $A(Q)=\left(\begin{array}{ll}x^{-3} & 0 \\ 0 & Y\end{array}\right)$ in $D=\{(X, Y): X>0, Y>0\}$, then $S$ is defined by $X^{-1}+Y^{-1}=1$.

We consider coordinates $r, Q=r^{-1} q$ to describe the behavior close to $q=0$, and $\rho=r^{-1}, Q=\rho q$ to describe behavior close to $q=\infty$. The corresponding momenta transformation makes the energy relation $H=h$ already regular.

i) For homogeneity $k<0$ of $U$ at $q=0$, let $Q=r^{-1} q, P=r^{-(\alpha+k) / 2} p$.

ii) For homogeneity $k>0$ of $U$ at $q=\infty$, let $Q=\rho q, P=\rho^{(\alpha+k) / 2} p$.

In the new coordinates $(r, Q, P)$ or $(\rho, Q, P)$ respectively, the energy relation $H=h$ becomes

$$
\begin{aligned}
& k<0: \frac{1}{2} P A^{-1}(Q) P^{t}=U(Q)+r^{|k|} h \\
& k>0: \frac{1}{2} P A^{-1}(Q) P^{t}=U(Q)+\rho^{|k|} h
\end{aligned}
$$

Consider the case $k>0$ at $q=\infty$. With a corresponding time rescaling $d t / d \tau=\rho^{-1+(k-\alpha) / 2}$, the equations (2) are defined and non trivial at $\rho=0$ :

$$
\begin{aligned}
& \rho^{\prime}=-\rho v, \\
& Q^{\prime}=P A^{-1}(Q)-v Q, \\
& P^{\prime}=\operatorname{grad} U(Q)-\frac{\alpha+k}{2} v P-\frac{1}{2} \frac{\partial}{\partial q}\left(P A^{-1}(Q) P^{t}\right),
\end{aligned}
$$


where the prime denotes derivative with respect to $\tau$,

$$
(1+\alpha / 2) v=P Q^{t}+\frac{1}{2} Q\left[\sum P A_{k}^{-1}(Q) \frac{\partial A}{\partial q_{k}}(Q)^{t}\right]
$$

and $A_{k}^{-1}(Q)$ denotes the $k-t h$ column of $A^{-1}(Q)$. In the new coordinates, the energy level $E_{h}=\{H=h\}$ extends to the following manifold with boundary

$$
\bar{E}_{h}=\left\{(\rho, Q, P): \rho \geq 0, Q \in S, \frac{1}{2} P A^{-1}(Q) P^{i}=U(Q)+\rho^{k} h\right\}
$$

Its boundary $N$, obtained by letting $\rho=0$ is called the infinity manifold. Since $\rho=0$ in system (3) implies $\rho^{\prime}=0$, it is clear that the extended vector feld is invariant on $N$.

Notice that the added boundary is common for any energy level $E_{h}$. This calls for an application of other blow ups to the boundary, in order to separate the different levels at $\rho=0$. This is not a simple task, but the first author has done it for the Kepler problem in a paper with G. Sienra [6].

We remark that if $h>0$, the study at $q=\infty$ for $k<0$ or at $q=0$ for $k>0$ can be made through a different blow up, not directly related to the homogeneity. For example, in the latter case we let $Q=r^{-1} q, P=r^{-\alpha / 2} p$, and the energy relation $H=h$ becomes $P A^{-1}(Q) P^{t}=2 r^{k} U(Q)+2 h$. In this case we already get different boundaries for each level $E_{h}$.

The case $\alpha=-2$, excluded in the above transformations car also be studied by means of a slightly different blow up. In this case the quantity $q A(q) q^{t}$ is homogeneous of degree 0 , and is not good for defining $r$ with the formula at the beginning of this section. However, notice that if $A(q)=|q|^{\alpha}$ Id where $|q|$ is the euclidean norm and Id is the identity matrix, we simply have for any $\alpha \neq-2$ that $r=\left(q A(q) q^{t}\right)^{\frac{1}{\alpha+2}}=|q|$. Hence, we find natural to define in this case $r=|q|$, even when $\alpha=-2$. For any admissible $A(q)$, we extend the definition $r=|q|$ for $\alpha=-2$. With the same definitions above for $Q$ and $P$, we get identical energy relations and time rescalings, only that the systern (3) may be slightly different. However, the flow in the case $\alpha=-2$ may show some undesirable behavior inexistent otherwise, as we will illustrate in Example 3, Section 4.

We will finish this section with a discussion of the blow up employed, so as to put it in the general context of blow ups.

We emphasize again that the blow up here is applied to the Hamiltonian function at $q=0$ (or at $q=\infty$ ), it is spherical and defined for $r \geq 0$ rather than projective, hence producing a boundary. In this scrise it is a little different from standard blow ups, which are not related to aryy finction.

Since blow up is performed on $H$, we have to consider its effect on each energy level $E_{h}$. Now $E_{h}$ in a neighborhood of $0 \in D$ exchuding 0 itself, is just a trivial fibration with fiber $S^{n-1}$. But excluding the origin is topologically equivalent 
to excluding a small closed ball $B$ about it. The effect of the blow up is to add $\partial B \cap D$, topologically equivalent to $S$. Since we are already in the non singular region, this boundary is assigned fibers $S^{n-1}$. This gives topologically $S \times S^{n-1}$ as the added boundary, which is in fact the divisor of the blow up.

In some cases we can take a double covering of the extended energy level $\bar{E}_{h}$, in such a way that $U$ extends naturally in a $C^{\infty}$ way. The divisor is the same as above, still an invariant submanifold but no longer a boundary. This is true in celestial mechanics where $k=-1, \alpha=0$ : taking $r<0$ can be interpreted as changing sign to $U$, getting a repulsive problem. However, this procedure is not valid whenever $r^{k}$ is not defined for $r<0$.

On the other hand, a projective blow up can be defined under more restrictive assumptions, since we would need $S$ to be symmetrical with respect to the origin, and agair $r^{k}$ has to be defined for $r<0$. Then we requirc to choose a way of identifying $S^{n-1}$ fibers at antipodal points of $S$.

\section{Behavior of extended flow}

In this section we study some important properties of the extended flow obtained after the blow up is applied. We assume that the blow up is as described in Section 2. An infinity manifold $N$ is defined through blow up at $q=\infty$ when $k>0$, while an origin manifold, denoted by $C$, is defined by blowing up at $q=0$ when $k<0$. This is understood in all the propositions, but the proofs and constructions are given in the former case, since the other case is similar. Proposition 4 asserts that with slight redefinition of variables, the blow up system (3) can be written in Hamiltonian form. The formula turns out to be different for $k=-2$, due to the fact that we have to integrate a power function. Let us remark that $k=-2$ gives in general the same sort of obstruction as $\alpha=-2$, discussed in the previous section.

The following result is a generalization of $[\mathbf{3}]$, for our case when the matrix $A$ is not constant.

Proposition 1. The infinity manifold $N$ (or the origin manifold $C$ ) is a $C^{\infty}$ manifold over the subset of $S$ where $\left.U\right|_{S} \geq 0$ whenever $O$ is a regular value of $\left.U\right|_{S}$.

Proof: Notice that for $\alpha \neq-2, N$ is diffeomorphic to $G^{-1}(0,0)$, where $G$ : $D \times \mathbb{R}^{n} \rightarrow \mathbb{R}^{2}$ is defined by $G(Q, P)=\left(Q A(Q) Q^{t}-1, P A^{-1}(Q) P^{t}-2 U(Q)\right)$. Then we have to check that the Jacobian matrix

$$
\left(\begin{array}{ll}
\operatorname{grad} Q A(Q) Q^{t} & , 0 \\
\operatorname{grad}_{Q} P A^{-1}(Q) P^{t}-2 \operatorname{grad} U(Q) & , 2 A^{-1}(Q) P^{t}
\end{array}\right)
$$

of $G$ has rank 2 on $G^{-1}(0,0)$. Indeed, if $U(Q)>0$ then $P \neq 0$ and the diagonal vectors in the above partition matrix are $\neq 0$, giving rank 2 . If on the 
other hand $U(Q)=0$, we necessarily have $P=0$, so that $\operatorname{grad}_{Q} P A^{-1}(Q) P^{t}=$ $A^{-1}(Q) P^{t}=0$. The only way for the rank to be 2 is that the vectors grad $Q A(Q) Q^{t}$ and $\operatorname{grad} U(Q)$ are non collinear on $S$, which means that 0 is a regular value of $\left.U\right|_{S}$.

For the remaining part of this section, we will assume that the mass matrix $A$ is constant. The following result, valid for any $k \neq-2$ is due to Devaney [3] when $k<0$. The forbidden value for $k$ will appear again in Proposition 4 .

Recall that a vector field $X$ on a manifold $M$ is almost gradient with respect to a function $f: M \rightarrow \mathbb{R}$, if $f$ always increases (or decreases) along non equilibrium solutions of $X$.

Proposition 2. Let the mass matrix $A$ be constant. Then the extended vector field on $N$ (or on $C$ ) is almost gradient with respect to $v$ ( $v$ increases along solutions if $k>-2$, and decreases if $k<-2$ ).

Let us just remark that the sharp change of behavior at $k=-2$ is due to the fact that $v^{\prime}=(1+k / 2) Q^{\prime} A Q^{\prime t}$ when $\rho=0$ (or $\left.r=0\right)$. In particular, if $k=-2$ solutions on the added boundary manifold are contained in the $v$-levels. If $k>0, v$ is always increasing along solutions on the infinity manifold $N$.

Next, we extend the concepts of homothetic solutions and central configurations, which are standard in celestial mechanics. Recall that if $A$ is constant, then system (2) is equivalent to the second order equation.

$$
\ddot{q} A=\operatorname{grad} U(q) .
$$

A solution of $(4)$ is called a homothetic solution if it is of the form $q(t)=\lambda(t) Q_{0}$, with $Q_{0} \in S$ a fixed vector. This leads us to the central configurations, since $Q_{0}$ must satisfy some conditions. Computing $\ddot{q}(t)$ and substituting into (4), we get

$$
\ddot{\lambda} Q_{0} A=\lambda^{d-1} \operatorname{grad} U\left(Q_{0}\right) .
$$

Multiplying now on the right by $Q_{0}^{t}$ and using the Euler formula for the homogeneity of $U$, we obtain the scalar second order equation

$$
\ddot{\lambda}=d \lambda^{d-1} U\left(Q_{0}\right)
$$

At the same time, we conclude that $Q_{0} A$ and grad $U\left(Q_{0}\right)$ are not only collinear vectors, but we have precisely

$$
\operatorname{grad} U\left(Q_{0}\right)=U\left(Q_{0}\right) d Q_{0} A .
$$

From Lagrange multipliers theory, this is equivalent to say that $Q_{0}$ is a critical point of $\left.U\right|_{S}$. We say that $Q_{0} \in S$ is a central configuration of the system if (5) is satisfied. 
Proposition 3. Let the mass matrix $A$ be constant. Then the homothetic solutions and possible equilibrium points of the flow on $N$ (or on $C$ ), are located by the critical points of $\left.U\right|_{S}$.

Proof: The assertion about homothetic solutions is clear from the above discussion. From the blow up equations (3), we see that the extended vector field has critical points when $\rho=0, P= \pm \sqrt{2 U(Q)} Q A$ and (5) holds. This means that they are on the infinity manifold $N$, the position vector $Q \in S$ is a central configuration, and $P$ has exactly two collincar values depending on $Q$. This requires of course $U(Q) \geq 0$.

Conditions for the existence of homothetic solutions are less stringent than conditions for existence of critical points on the infinity manifold. For a thorough discussion of this point, see [5].

Now, because of the homogeneity, it is possible to show that with a slight redefinition of variables, the blow up vector field can be put in Hamiltonian form. This requires an insight on the structure given by the canonical simplectic form $\omega=d p \wedge d q$ of the phase space $T^{*} D=D \times \mathbb{R}^{n}$, by applying techniques of contactization and symplectization. See [5] for details, but the conclusion can be formulated as follows, if we define a new momentum and radial coordinate for $k \neq-2$ by

$$
\bar{P}=\rho^{-1-k / 2} Q^{\prime} A, \quad \lambda=\rho^{-1-k / 2} /(1+k / 2) .
$$

Proposition 4. Let the mass matrix $A$ be constant and $k \neq-2$. Then the change of coordinates from $(p, q)$ to $(Q, \lambda, \bar{P}, v)$ is canonical. The same thing for $k=-2$ if we redefine $\lambda=-\ln \rho$.

Proof: We just have to verify that the symplectic form $\omega=d p \wedge d q$ becomes $d \bar{P} \wedge d Q+d v \wedge d \lambda$ in the new coordinates. Indeed, we have $p d q=$ $\rho^{-k / 2} p d\left(\rho^{-1} Q\right)=\rho^{-2-k / 2}\left(-\left(v+Q^{\prime} A Q\right) d \rho+\rho Q^{\prime} A d Q\right)=v d \lambda+\bar{P} d Q$, where we used $P=v Q A+Q^{\prime} A$ and the fact $Q^{\prime} A Q=0$ since $Q \in S$ with $A$ constant. Applying exterior differentials, we are done.

The procedure for going from Equations (2) to the blow up equations (3), will be to transform the Hamiltonian $H$ by the above canonical transformation, writing then the corresponding Hamilton equations. We make the change of time scale $d t / d \tau=\rho^{-1+(k-\alpha) / 2}$, converting finally to $(\rho, Q, P)$ coordinates where $P=\left(Q^{\prime}+v Q\right) A$, with the help-of $(6)$. 


\section{Detailed examples}

In this section we analyze the result of applying blow up transformations to 4 examples.

In any case, we see from (1) that $E_{h}=\{H=h\}$ is a pinched $S$-bundle over the Hill region $\{q \in D: U(q)+h \geq 0\}$, in Smale's terminology. This means that the fiber is pinched to a point on the boundary $U+h=0$.

1) Anisotropic Kepler problem (see [3]). This is a generalization of the Kepler problem in celestial ruechanics. Its Hamiltonian can be written as

$$
H\left(q_{1}, q_{2}, p_{1}, p_{2}\right)=\left(p_{1}^{2}+p_{2}^{2}\right) / 2-\left(\gamma^{2} q_{1}^{2}+q_{2}^{2}\right)^{-1 / 2},
$$

where $\gamma \geq 1$, and $\gamma^{2} q_{1}^{2}+q_{2}^{2} \neq 0$. If $\gamma=1$ we have the standard Kepler problem, but whenever $\gamma>1$, it becomes a no longer integrable problem. A further thorough study of the possible motions using symbolic dynamics has been made in [2].

In this case the metric is the euclidean one in the plane. The set $\{U+h \geq 0\}$ is topologically a punctured closed disk if $h<0$ or the punctured plane (no restriction) otherwise. This mears that there is no infinity manifold in the former case.

We show $E_{h}$ in figure 1, with its infinity manifold $N$ and the corresponding boundary obtained by blow up at the origin (denoted by $C$ ). All the figures have to be idertified at the right and left ends, since the horizontal coordinate is the polar angle in configuration plane.

There are 4 central configurations corresponding to the positive and negative directions of the two axes. Hence, there are exactly 8 equilibriums points on $C$ for any $h$ and on $N$ for $h=0$. An exception is the infinity manifold $N$ for $h>0$, which has two circles of equilibrium points shown at the top and the bottom of Fig. 1, c. Homothetical solutions inside the figures are shown with upward vertical arrows.

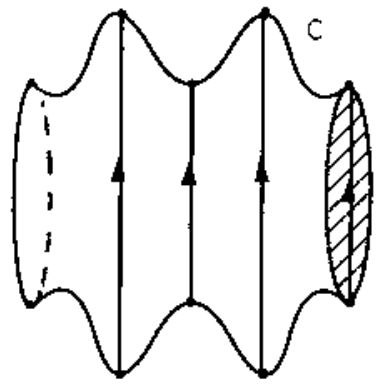

a) $h<0$

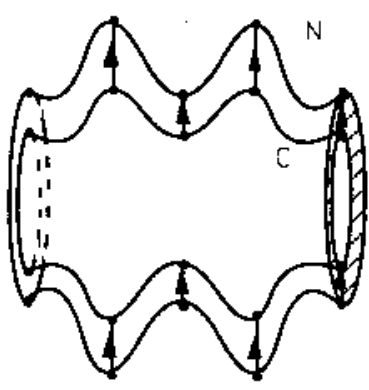

b) $h=0$

Figure 1

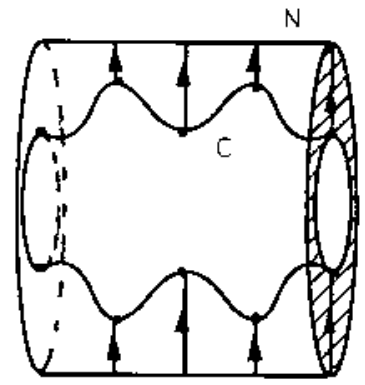

c) $h>0$

The shape of the toral surfaces is due to the variation of $\left.U\right|_{S}$. In particular 
if $\gamma=1$, then $\left.U\right|_{S}$ is constant and we get standard tori.

2) System with a Henon-Heiles potential. We consider here a mechanical system with a homogeneous Henon-Heiles potential [1]. In this case $k=3$, since

$$
H\left(q_{1}, q_{2}, p_{1}, p_{2}\right)=\left(p_{1}^{2}+p_{2}^{2}\right) / 2+\alpha\left(q_{1} q_{2}^{2}-q_{1}^{3} / 2\right) .
$$

There is an equilibrium point in phase space: the origin located in the energy level $h=0$. To see that, we write explicity Hamilton equations:

$$
\dot{q}_{1}=p_{1}, \dot{q}_{2}=p_{2}, \dot{p}_{1}=\left(3 q_{1} / 2-q_{2}^{2}\right) \alpha, \dot{p}_{2}=-2 \alpha q_{1} q_{2}
$$

Hill regions for the 3 possible cases are shown in Fig. 2. We have again the euclidean metric in $\mathbb{R}^{2}$. By applying Lagrange multipliers, it is easy to check that there are six central configurations corresponding to the vertices of the hyperbolas. This is intuitively clear from the figures, by recalling the interpretation of central configurations as critical points of $U$ restricted to the unit sphere.

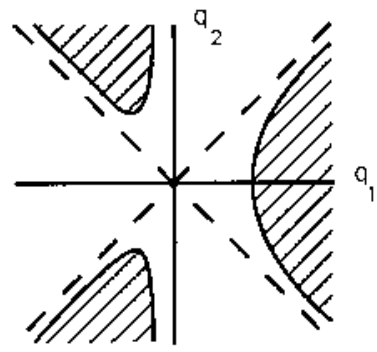

a) $h<0$

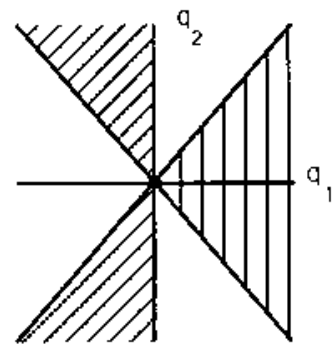

b) $h=0$

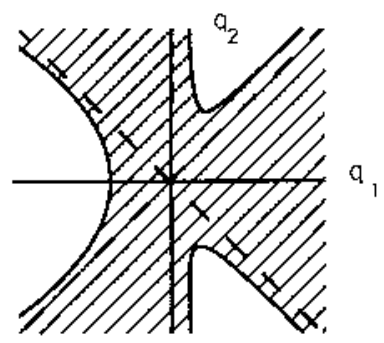

c) $h>0$

Figure 2

In Fig. 3 wee depict topologically $E_{h}$ with origin and infinity manifolds for $h \neq 0$. We remark that there are three components and no origin manifold for $h<0$, as can be verified from Fig. 2. If $h>0$ the figure has to be identified as in Example 1. The origin manifold $C$ has one component but 2 circles of equilibria, as manifold $N$ in Fig. 1, c.

Homothetical solutions are shown as in the other example. We see that the number of critical points on $N$ does not double the number of central configurations as in Example 1, since escape to infinity is forbidden in many directions. 

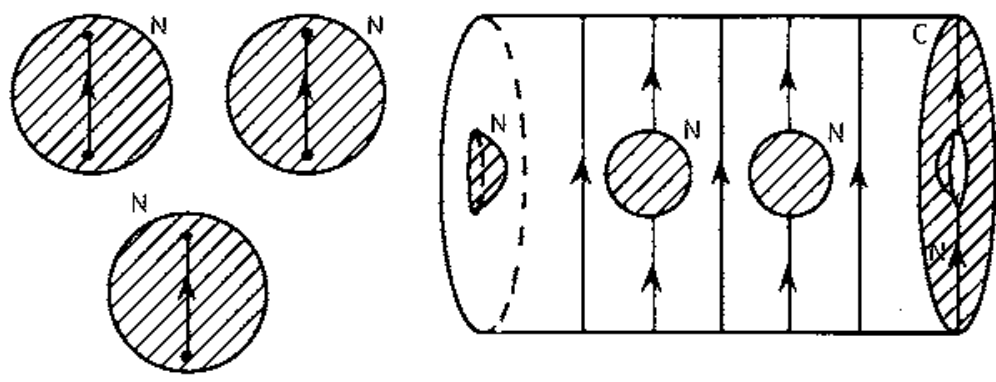

Figure 3

3) Central potential with conformal metric. We consider here a mechanical system in $\mathbb{R}^{n}$, with a homogeneous central potential and a conformal metric $d s^{2}=R^{\alpha}\left(\sum d q_{i}^{2}\right)$, where $R^{2}=q_{1}^{2}+\cdots+q_{n}^{2}$. This is a generalization of Example $C$ in Section 1 . The Hamiltonian has the form

$$
H(q, p)=R^{-\alpha}|p|^{2} / 2-R^{k},
$$

and we will assume that $k \leq 0$. In this case, $A=R^{\alpha}(\mathrm{Id})$ while $r=R$ is the euclidean norm. Let us consider the blow up equations at $q=0$, where the potential becomes singular (if $k<0$ ) and the kinetic energy degenerates to zero if $\alpha<0$. With $P=r^{(\alpha+k) / 2} p$, the blow up equations are

$$
\begin{aligned}
& r^{\prime}=r(P \cdot Q) \\
& Q^{\prime}=P-(P \cdot Q) Q \\
& P^{\prime}=\left(\alpha|P|^{2} / 2+k\right) Q-1 / 2(\alpha+k)(P \cdot Q) P,
\end{aligned}
$$

where the change of time scale is $d t / d \tau=r^{1+(\alpha-k) / 2}$ and the energy relation is $|P|^{2} / 2-1=r^{-k} h$. Setting $r=0$, we get the origir manifold as

$$
C=\left\{\left.(Q, P)\left|Q \in S^{n-1}, \quad\right| P\right|^{2}=2\right\},
$$

topologically equivalent to $S^{n-1} \times S^{n-1}$. The equilibrium points of the extended flow are defined by $r=0, P= \pm \sqrt{2} Q$, representing two disjoint copies of $S^{n-1}$ in $C$.

If $k=0$, we compute easily the eigenvalues at any equilibrium point as $u_{0}=P_{0} \cdot Q_{0}$ with multiplicity $I$ in the radial direction, 0 with multiplicity $n$ including a transversal direction to $E_{h}$, and $-(1+\alpha / 2) z_{0}$ with multiplicity $n-1$. Thus, the flow is normally hyperbolic at the equilibrium submanifold, provided that $\alpha \neq-2$. We recall that $\alpha=-2$ is in general a problematic case, as we remarked in Section 2.

4) Hyperbolic metric in the plane. Recall that this is $d s^{2}=y^{-2}\left(d x^{2}+\right.$ $\left.d y^{2}\right)$, so that the Hamiltonian is $H(q, p)=y^{2}|p|^{2} / 2$ where $q=(x, y), p=$ $\left(p_{1}, p_{2}\right)$ and the corresponding Hamilton equations read as

$$
\dot{p}_{1}=0, \dot{p}_{2}=-y|p|^{2}, \dot{x}=y^{2} p_{1}, \dot{y}=y^{2} p_{2} .
$$


This is the special case $\alpha=-2$, and in addition we have a singularity at the whole $x$-axis and not only at the origin. Hence, we consider a slight variation (otherwise very natural) of the transformation given for $\alpha=-2$, to blow up the whole axis. We check that since $k=0$, no change of time scale is needed.

Let $P=y p$, then the equations of motion become

$$
\begin{aligned}
& \dot{x}=y P_{1} \\
& \dot{y}=y P_{2} \\
& \dot{P}_{1}=P_{1} P_{2} \\
& \dot{P}_{2}=-P_{1}^{2} .
\end{aligned}
$$

The last 2 equations are uncoupled from the other two, so that the system can be integrated by quadratures. If $P_{1}=0$, we get $x=$ constant and $P_{2}=$ constant, which are the vertical lines. If $P_{1} \neq 0$, we rescale the time by $d t / d \tau=$ $P_{1}^{-1}$, getting the circles

$$
P_{1}(\tau)=\sqrt{2 h} \cos \tau, P_{2}(\tau)=\sqrt{2 h} \sin \tau, x(\tau)=B \sin \tau+a, y(\tau)=B \cos \tau
$$

where $B$ and $a$ are constants:

The blow up produces the following extended energy level, for any energy $h>0$ :

$$
\bar{E}_{h}=\left\{\left(x, y, P_{1}, P_{2}\right): y \geq 0, x \in \mathbb{R}, P_{1}^{2}+P_{2}^{2}=2 h\right\}
$$

which topologically is $\mathbb{R} \times\left(\mathbb{R}^{+} \cup\{0\}\right) \times S^{1}$. By setting $y=0$, we get a boundary manifold $C$ invariant under the extended flow, which topologically is a cylinder. It has 2 lines of critical points as in Examples 1 and 2.

We observe that if we define $\lambda=x / y$, an analog of the $Q$-variable in the general theory of Section 2, the equations (9) become

$$
\begin{aligned}
& \dot{y}=y P_{2} \\
& \dot{\lambda}=P_{1}-\lambda P_{2} \\
& \dot{P}=P_{2} P+\left(0,-|P|^{2}\right) .
\end{aligned}
$$

This system is remarkably similar to (3). Besides, the equation $\dot{P}_{2}=-P_{1}^{2}$ in (9) implies that the flow on $C$ is almost gradient with respect to $P_{2}$.

If we change the variable $y$ by $\rho=y^{-1}$ with a change of time scale $d t / d s=$ $\rho^{-1}$, we get

$$
\begin{array}{ll}
x^{\prime}=P_{1} & P_{1}^{\prime}=\rho P_{1} P_{2} \\
\rho^{\prime}=-\rho^{2} P_{2} & P_{2}^{\prime}=-\rho P_{1}^{2} .
\end{array}
$$

These new coordinates produce another boundary $N$ on the $y \rightarrow+\infty$ side of the half-plane when we let $\rho=0, x \in \mathbb{R}, P_{1}^{2}+P_{2}^{2}=2 h$, which topologically is another cylinder. 
The projection of the flow in $E_{h} \cup C \cup N$ into a plane where $\left(P_{1}, P_{2}\right)$ locates the direction and the radial distance is $(3 y+1) /(y+1)$, can be described as in Fig. 4. The flow on $N$ is by horizontal lines transversal to the figure, except at the two lines $P_{1}=0$ of critical points.

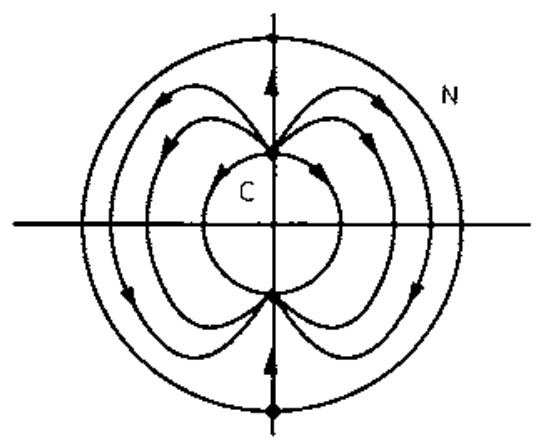

Figure 4

Acknowledgements. One of us (EAL) acknowledges insights into the nature of blow ups from Felipe Cano (Spain).

\section{References}

1. R. BROUCKE, "Simple non integrable systems with two degrees of freedom," Instabilities in Dynamical Systems (V. Szebehely, Ed.), Reidel Publ., 1979.

2. J. Casasayas and J. Llibre, Qualitative analysis of the anisotropic Kepler problem, Memoirs Amer. Math. Soc. 312 (1984).

3. R. Devaney, "Singularities in classical mechanical systems," in Ergodic Theory and Dynamical Systems I (A. Katok, Ed.), Birkhauser, Basel, 1981, p. 211.

4. E. LaCOMBA, Blow up on energy levels in celestial mechanics, Publicacions Secció de Matemàtiques, Univ. Autónoma de Barcelona 28 (1984), 97-117.

5. E. LACOMBA AND L. IBORT, Origin and infinity manifolds for mechanical systems with homogeneous potentials, Acta Appl. Math. 11 (1988), 259-284. 
6. E. LaCOMBA AND G. SienRA, "Blow up techniques in the Kepler problem," Holomorphic dynamics (Gómez-Mont, Seade, Verjovsky, eds.) Springer-Verlag Lect. Notes 1345, 1988, p. 177.

7. E. LACOMBA AND C. Simó, Boundary manifolds for energy surfaces, $\mathrm{Ce}$ lestial Mechanics 28 (1982), 37-48.

8. R. MCGEHEE, Triple collision in the collinear three body problem, Inventiones Math. 27 (1984), 191 -227.

Ernesto A. Lacomba: Departamento de Matematicas

Universidad Autónona Metropalitana

Unidad Iztapalapa, Apartado Postal 55-534

C.P. 09340 MÉXICO D.F.

John Bryant: 47 Ave. Felix Fauré

75015 Paris

FRANCE

Iuis A. Ibort: Department of Mathemalics

University of California at Berkeley

Berkelcy, Cal. 94720

U.S.A.

Rebut el 21 de Març de 1990 